\title{
Averting the gaze disengages the environment and facilitates remembering
}

\author{
ARTHUR M. GLENBERG, JENNIFER L. SCHROEDER, and DAVID A. ROBERTSON \\ University of Wisconsin, Madison, Wisconsin
}

\begin{abstract}
When people are asked moderately difficult questions, they often avert their gazes. We report five experiments in which we documented this phenomenon. They demonstrate that (1) the frequency of gaze aversion is related to the difficulty of cognitive processing, (2) this behavior cannot be due solely to demand characteristics or embarrassment, and (3) the behavior is functional: Averting the gaze improves performance. We speculate that averting the gaze helps people to disengage from environmental stimulation and thereby enhances the efficiency of cognitive processing directed by nonenvironmental stimulation.
\end{abstract}

There is a secret bond between slowness and memory, between speed and forgetting. Consider this utterly commonplace situation: a man is walking down the street. At a certain moment, he tries to recall something, but the recollection escapes him. Automatically, he slows down.

$$
\text { -Milan Kundera, Slowness (1996, p. 39) }
$$

What is behind Kundera's commonplace observation? Our research suggests an answer: When engaged in difficult cognitive activity, we close our eyes or look at the sky to suppress the environment's control over cognition. Because information from the environment is suppressed, we slow down and stop walking to avoid hurting ourselves. In the five experiments reported here, we document behaviors associated with moderately difficult remembering - namely, averting the gaze by closing the eyes, covering the eyes, or looking at a uniform field such as the floor, ceiling, or sky. In Experiments 1 and 2, we demonstrate that the frequency of gaze aversion is correlated with difficulty of the autobiographical (Experiment 1) or general knowledge (Experiment 2) questions asked. In Experiment 3, we showed that people avert their gaze even when social cues are eliminated. Experiments 4 and 5 demonstrate that this form of behavior is functional; that is, the behavior is causally related to improvements in performance.

Why might people engage in gaze aversion? The phenomenon is predicted from an analysis of memory reported in Glenberg (1997). Glenberg proposes that memory evolved in the service of action, and that potential patterns of action are the basis of meaning and conceptualization.

This work was supported in part by AFOSR AASERT Grant F4962092-J-0310 and University of Wisconsin Graduate Research Committee Grant 940109. The data were presented at the meeting of the Psychonomic Society in 1995, and they serve as part of the second author's senior honor's thesis. We thank Jennifer Jansen for her help in collecting the data for Experiment 4, and Dacher Keltner for the use of his equipment and laboratory space for Experiment 3 . Requests for reprints should be sent to A. M. Glenberg, Department of Psychology, 1202 West Johnson Street, Madison, WI 53706 (e-mail: glenberg@facstaff.wisc.edu).
Furthermore, action must respect constraints of the environment, or we would soon find ourselves falling off of cliffs, walking into rivers, or colliding with automobiles. To guarantee that environmental constraints are respected, the cognitive system is normally "clamped" to the environment. That is, environmental constraints on action normally control conceptualization (the pattern of actions currently available). Ballard, Hayhoe, Pook, and Rao (1997) have developed a similar analysis: pointing with the eyes (deictic eye movement) binds environmental objects to variables in cognitive programs, thereby allowing characteristics of the environment to control cognition. Nonetheless, many significant human activities, such as planning, conscious recollection, and language comprehension, require that we disengage from the environment. For example, recollected events may have little to do with the physical environment in which they are recollected. Thus, if the environment retained sole control over conceptualization as it may with some animal species (cf. Gärdenfors, 1996), recollection would be impossible, or at least severely limited. Finally, disengaging or suppressing the environment's control over conceptualization may be effortful. The effort assures that whenever we are disengaged from the environment we are consciously aware of that fact, and we do not take precipitous action on the basis of imagination (e.g., acting on the imagined ability to leap tall buildings or run faster than a speeding bullet), nor do we (or Kundera) continue to walk without looking where we are going.

If disengaging from the environment is cognitively effortful, then gaze aversion is well motivated: The physical effort of averting the gaze (and thereby reducing environmental stimulation) may be much less demanding than the attempt to cognitively suppress the environment's control over conceptualization. Furthermore, the overt behavior should be related to the difficulty of the cognitive task. Some cognitive tasks may be completed relatively automatically, and in fact, some may be facilitated by environmental stimulation. An important class of such tasks comprises object recognition, naming, and classification. 
These tasks are facilitated by focusing on the cues that the environment provides rather than by ignoring the cues. However, to the extent that tasks are more conceptually driven (Roediger, Guynn, \& Jones, 1994) than data driven, and to the extent that tasks are cognitively difficult, we should see an increase in gaze aversion. These predictions are also consistent with an account of remembering as a dual-task situation. ${ }^{1}$ This account is discussed in General Discussion.

The behavior of averting the gaze has been reported previously, perhaps most often in the guise of lateral eye movements, or LEMs (see, e.g., Kinsbourne, 1972). The phenomenon was thought to be significant because initial work seemed to demonstrate that the lateral direction of the eye movement was contralateral to the cerebral hemisphere activated by a cognitive task. Thus verbal information processing, which supposedly activates the left hemisphere, produces rightward eye movements, and spatial information processing, which supposedly activates the right hemisphere, produces leftward eye movements. There appear to be a number of problems with this hypothesis, however, as has been documented by Ehrlichman and Weinberger (1978) in a literature review, as well as by others in subsequent experimental reports and reviews (e.g., De Gennaro \& Violani, 1988). Primary among these problems are difficulties in replicating the laterality effect: Fewer than half of the 79 experiments summarized by De Gennaro and Violani produced the predicted pattern of LEMs. The direction of eye movements appears to be affected by many factors unrelated to the type of question asked, including the position of the experimenter, the presence of a video camera, and the mode of participants' responses (Ehrlichman \& Weinberger, 1978; Kinsbourne, 1974; Richardson, 1978). Also troubling for the laterality hypothesis is the finding that many eye movements occur in the vertical plane rather than the horizontal plane. These vertical movements do not correspond to predictions based on cerebral laterality.

Interestingly, although the laterality hypothesis provided the impetus for much of this research, several investigators have noted the possibility of a connection between difficulty of the cognitive processing and ocular motility (the frequency of eye movements). Among the first to note this possible relation was Day (1964), although he reported no data. Meskin and Singer (1974) noted that questions that presumably demanded extensive memory search (e.g., "Describe how Lee Harvey Oswald was shot?") generated greater motility than did questions that presumably required less search of memory (e.g., "How old are you?"). Unfortunately, there did not appear to be any objective measure of difficulty or of the extent of memory search. Also, there did not appear to be any control over the time taken to answer the question and hence the time available to observe an eye movement. De Gennaro and Violani (1988) used questions rated as hard or easy and noted greater motility with the harder questions. Again, there did not appear to be any control over the time available for observing eye movements.
In the experiments reported here, we examined a related question: Do we avert our gaze from areas of stimulation (not just move the eyes in any direction) in order to facilitate cognitive processing? We will demonstrate that people do avert the gaze frequently for questions of moderate difficulty. Also, we will demonstrate functional consequences: Averting the gaze improves performance.

\section{EXPERIMENT 1}

In describing this experiment, we introduce the basic procedure and demonstrate the effect in an autobiographical memory task. Participants were asked to answer a series of questions such as "What did you have for breakfast today?" We manipulated the difficulty of the question by the retention interval queried. Thus, the question could be changed to, "What did you have for breakfast yesterday?" Each question was printed on an index card and shown to the participant. After a 10 -sec interval, a signal indicated that the participant should answer the question or say "I don't know." The purpose of this interval was to enforce a constant amount of time during which the participant could engage in averting the gaze, regardless of question difficulty. Otherwise, because easy questions probably take less time to answer than difficult questions, the amount of time during which participants could avert their gaze would be confounded with the difficulty of the question. During the 10-sec interval, the experimenter noted (unbeknownst to the participant) whether or not the gaze had been averted, and she recorded the participant's answer at the end of the interval.

\section{Method}

Participants. The participants were 19 students enrolled in introductory psychology classes at the University of Wisconsin, Madison. They earned credit toward a research requirement by participating in the experiment.

Materials. We constructed 30 questions, 3 in each of 10 domains. The 3 questions in each domain varied in the duration of the retention interval queried (short, intermediate, and long). For example, 3 questions in 1 domain asked the participant to name a current professor, one from a semester ago, and one from two semesters ago. The 30 questions were each printed on an index card. Because of a printing error that resulted in the repetition of 1 question, all analyses will focus on 27 questions from 9 domains. The cards were randomized for each participant under the constraint that each third of the order contained 1 question from each of the 10 domains.

Procedure. After written consent had been obtained, each participant was told that the experiment concerned memory for autobiographical events, and that he/she would be asked to answer a series of 30 questions. The experimenter sat across from the participant, approximately $1.5 \mathrm{~m}$ away. The participant read each question silently from the index card held by the experimenter. The silent reading of the question, along with the randomization of the cards, ensured that the experimenter was blind to the retention interval condition. The experimenter started a timer simultaneously with the exposure of the card. After a 10-sec interval, the timer generated an auditory alarm that signaled the participant to respond with the answer to the question or with the phrase "don't know." During the interval, the experimenter noted whether the participant averted his/her gaze from the direction of the experimenter and the index card. A gaze aversion was scored if the participant looked toward 
the floor, wall, or ceiling with an eye or a head movement, or if the participant closed or covered the eyes. The experimenter also recorded the participant's answer. The main series of 30 questions was preceded by 3 practice questions.

\section{Results and Discussion}

The proportions of times (i.e., trials on which) participants averted their gaze were $.37, .40$, and .53 for the short, intermediate, and long retention intervals, respectively. The increase in gaze aversion over the retention interval was statistically significant [using a probability of a Type I error of .05 for all analyses; $F(2,36)=4.39$, $\left.M S_{\mathrm{e}}=.032\right]$. The contrast between the short and intermediate intervals was not significant $[F(1,18)=1.62]$, but the contrast between the short and long intervals was significant $\left[F(1,18)=6.71, M S_{\mathrm{e}}=.04\right]$, as was the contrast between the intermediate and long intervals $[F(1,18)=$ 6.32, $\left.M S_{\mathrm{e}}=.02\right]$.

These results illustrate that people frequently avert their gaze. Also, this behavior increases with the difficulty of the cognitive task. (An index of difficulty is the proportion of "don't know" answers. That proportion increased from .06 to .11 to .22 across the retention intervals.)

\section{EXPERIMENT 2}

We extended the phenomenon in two ways in Experiment 2 . First, instead of questions querying autobiographical memory, we asked general knowledge questions. Second, because the general knowledge questions had objective answers, we could use proportion correct rather than proportion "don't know" as an index of difficulty. Other than the use of general knowledge questions, the method was substantially the same as that of Experiment 1 .

\section{Method}

Participants. The 18 participants were students enrolled in introductory psychology classes at the University of Wisconsin, Madi- son, who earned credit toward a research requirement by participating in the experiment. One participant was eliminated because she was a non-native English speaker, and during the debriefing she indicated that she had problems in generating the answers in English.

Materials. We selected general knowledge questions from Nelson and Narens (1980). Our intent was to select questions with a proportion of correct answers in the range of 70 to 1.00 . We did not wish to ask more difficult questions for the following reason. People can fairly quickly and accurately judge whether or not they know the answer to a question, and that judgment is often made on the basis of the familiarity of the terms in the question (Reder \& Ritter, 1992; Schwartz \& Metcalfe, 1992). Thus, although some questions can be quite difficult as measured by proportion correct, in fact those questions may not engender extensive cognitive activity. Because we are predicting an increase in the frequency of averting the gaze with an increase in cognitive activity rather than an increase in question difficulty as measured by accuracy, we decided to limit the range of accuracies to 70 to 1.0 . We selected 30 questions from this range. Three of the questions were used for practice, and the remaining 27 were used in the experiment proper.

\section{Results and Discussion}

Contrary to expectations based on the Nelson and Narens (1980) norms, only 9 of the 27 questions were answered with a proportion correct greater than .7. For those 9 questions, the relation between difficulty and proportion of times that participants averted their gaze is illustrated in Figure 1. The correlation between these two variables, -.83 , is statistically significant. ${ }^{2}$

These data confirm the conclusions drawn from Experiment 1 and extend them to the domain of general knowledge questions. People avert the gaze when answering questions, and the frequency of gaze aversion is correlated with question difficulty (i.e., with cognitive effort).

\section{EXPERIMENT 3}

There are two problems with the demonstrations thus far. First, the relationship between question difficulty and averting the gaze was demonstrated for only a small

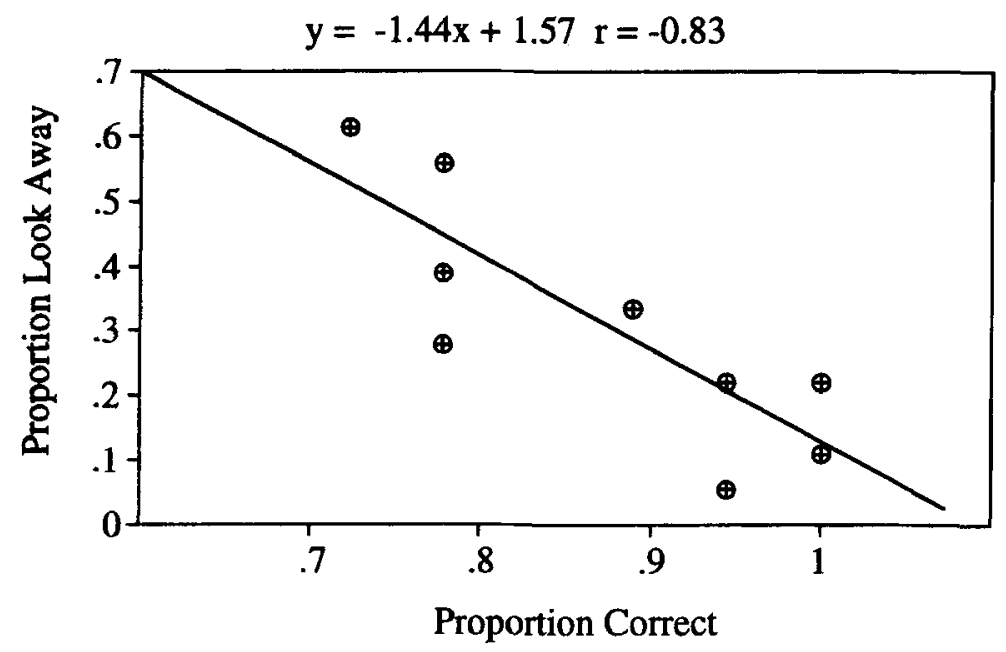

Figure 1. The data are from Experiment 2. Each symbol corresponds to a single question. 
number of questions in Experiment 2. Thus, in Experiment 3 , we increased the number of questions asked by selecting new questions from the upper range of the Nelson and Narens (1980) norms. Second, the possibility remained that the effect was produced more by embarrassment or by task demands than by any effort to disengage from the environment. Suppose that the participants believed that we were asking questions for which they should have known the answers. If the answer was not forthcoming (that is, if it was a difficult question), the participant might become embarrassed by the awkward social situation and this embarrassment would be the cause of averting the gaze (Argyle \& Kendon, 1967). We attempted to obviate this social explanation by taking two steps. First, we presented the questions to the participants on a computer screen. Second, the experimenter was not present while the participant answered the questions. Instead, the participant's behavior was videotaped by a hidden camera. Videotaping also allowed us to check the reliability of the coding of gaze aversion by having multiple people code the gaze aversion recorded on videotape.

\section{Method}

Participants. The 17 participants were students enrolled in introductory psychology classes at the University of Wisconsin, Madison, who earned credit toward a research requirement by participating in the experiment.

Materials. We began with 40 questions: 27 new ones from the Nelson and Narens (1980) norms, and the 13 questions from Experiment 2 with the highest accuracies (including practice questions). After pilot testing, we eliminated 10 of these 40 questions. The remaining 30 questions all had norms of .60 or above and included the 9 questions analyzed in Experiment 2 .

Procedure. The procedure was very similar to that of Experiment 2 except for the following changes. The questions were presented on a computer screen. At the onset of the question, the computer played a single short tone (to signal the beginning of the observation period when the video tapes were scored), and after the delay of $10 \mathrm{sec}$ the computer played two short tones to signal that the participant should respond. Responding consisted of typing an answer on the computer keyboard. The participants' behavior was monitored by a hidden camera. Although the consent form indicated that the participant might be videotaped, this was not mentioned in the instructions to the participant.

\section{Results and Discussion}

Two people independently scored gaze aversion. The agreement between them was $95 \%$. When the two disagreed, we used the scoring produced by the person who had scored behavior in the previous experiments. One participant's data were eliminated from further consideration. That participant's mean proportion correct $(.27)$ was 2.94 standard deviations below the mean for all 17 participants (.80). After we had eliminated questions with accuracies less than .70 , we were left with 23 questions. The relation between question answering accuracy and the proportion of times that participants averted their gaze is portrayed in Figure 2. The correlation between the two variables, -.55 , is statistically significant. ${ }^{3}$

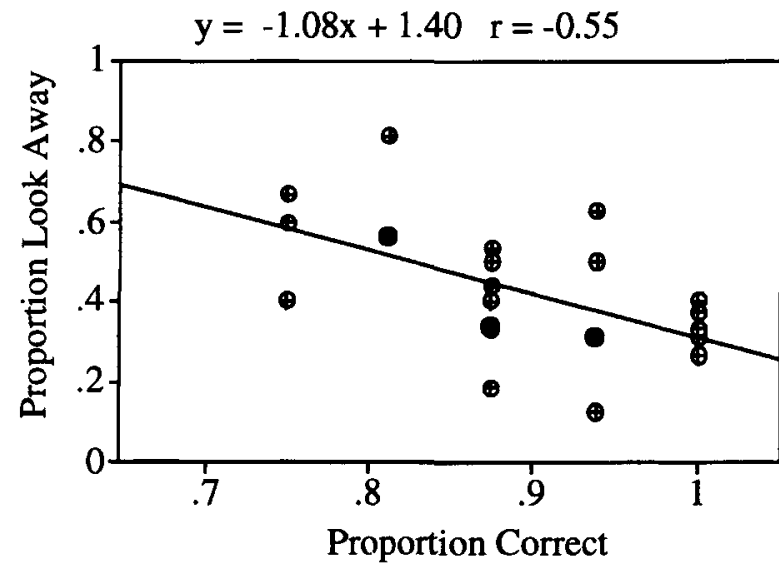

Figure 2. The data are from Experiment 3. The open symbols correspond to single questions, and the solid symbols indicate overlapping data from two questions.

These results add to our confidence in the effect in three ways. First, we replicated the effect with a wider range of questions. Second, we demonstrated that the coding of averting the gaze was reliable. Third, because there was no one in the experimental room with the participant, it is unlikely that averting the gaze was produced solely by embarrassment.

\section{EXPERIMENT 4}

According to the framework developed in the introduction, averting the gaze is functional. That is, disengaging from the environment facilitates internal control over conceptualization and should thus increase accuracy. Unfortunately, the data from Experiments 1-3 cannot be used to test this claim. We cannot score accuracy of the idiosyncratic answers given in Experiment 1. One might suppose that we could test the claim by using the data from Experiments 2 and 3: When participants chose to look away they should have been more accurate. Unfortunately, if the framework is correct, then looking away is an index of difficulty. That is, participants choose to look away when the question is particularly difficult for them. Although looking away should then help, there is no guarantee that it will overcome the extra difficulty of that question for that participant. Instead, one must manipulate the opportunity to look away.

In this experiment, we directly manipulated whether or not participants could avert their gaze. In one condition (close eyes), participants were instructed to close their eyes in the interval between reading the question and the occurrence of the auditory signal. In the other condition (look), they were instructed to look at the experimenter's nose. These conditions were manipulated within subjects in counterbalanced order. In addition, to extend the range of materials that we examined for the effect, we manipulated (within subjects) the type of question 
asked. The 30 critical general knowledge questions from Experiment 3 were used. Participants were also asked to solve 30 simple mathematics questions. Finally, we manipulated (within subjects) question difficulty (easy, medium, or difficult). The prediction was that for the moderately difficult questions, subjects would perform better in the close eyes condition than in the look condition.

\section{Method}

Participants. The 29 participants were members of the University of Wisconsin summer community recruited through various campus and newspaper advertisements as well as from an introductory psychology class. The students from the class received credit toward a research requirement for participating; the others received a cash payment. The data from 1 subject were eliminated because excessive construction noise outside of the experimental room seemed to be quite distracting to that person.

Materials. The 30 general knowledge questions were taken from Experiment 3. They were rank ordered on the basis of performance in an unreported experiment, and then the members of each successive pair of questions were randomly assigned to two sublists.

We also generated 30 mathematics questions. The 10 "easy" questions were of the form $w+x+y=z$. The sum, $z$, was a two-digit number, and one of the addends was designated by a question mark. The participants' task was to state a number with which to replace the question mark so that the equation would be correct. The 10 "moderate" questions and the 10 "difficult" questions took the form of $x / y=$ ? or $x * y=$ ? The size of $x$ and $y$ were adjusted to make the questions more or less difficult. The 30 questions were randomly assigned to two sublists under the constraint that each sublist contain 5 easy, 5 moderate, and 5 difficult questions.

One sublist of 15 mathematics questions was randomly combined with one sublist of general knowledge questions under the constraint that groups of 6 successive questions contain 3 general knowledge questions ( 1 at each level of difficulty) and 3 mathematics questions ( 1 at each level of difficulty). This was also done for the other sublists of questions. These two sets of 30 questions were assigned to the close eyes and look conditions in a counterbalanced fashion.

Procedure. The procedure was similar to that of Experiments 1 and 2. Participants practiced on 2 general knowledge and 2 mathematics questions. Each question was presented on an index card held by the experimenter, who also started a timer. After a 10 -sec interval, the timer sounded and the subject was to produce an answer. Immediately before each block of 30 questions ( 15 general knowledge and 15 mathematics questions), participants were instructed to either close their eyes during the interval or look at the experimenter's nose. The experimenter recorded the answer and noted whether or not the participant complied with the instructions.

\section{Results and Discussion}

Across the 28 participants, 1,680 questions were asked. The participants complied with the instructions on 1,656 of these, for a compliance rate of .99 .

The predictions apply to the questions of moderate difficulty. Because the participants' mathematical skills differed dramatically, the following procedure was used for each participant in order to obtain a set of subject-defined medium difficulty items. First, we considered general knowledge and mathematics questions separately. Second, we collapsed across the close eyes and look conditions. Third, we rank ordered the mean correct for the three nominal difficulties (easy, moderate, and difficult). We chose the subject-defined medium difficulty items to be the 10 items in the middle of the rank order. Fourth, we computed the mean correct for the close eyes and look conditions for further analyses.

The prediction is that performance would be greater in the close eyes condition than in the look condition. The relevant data, presented in Figure 3, confirm this prediction.

\section{Moderately Difficult Items}

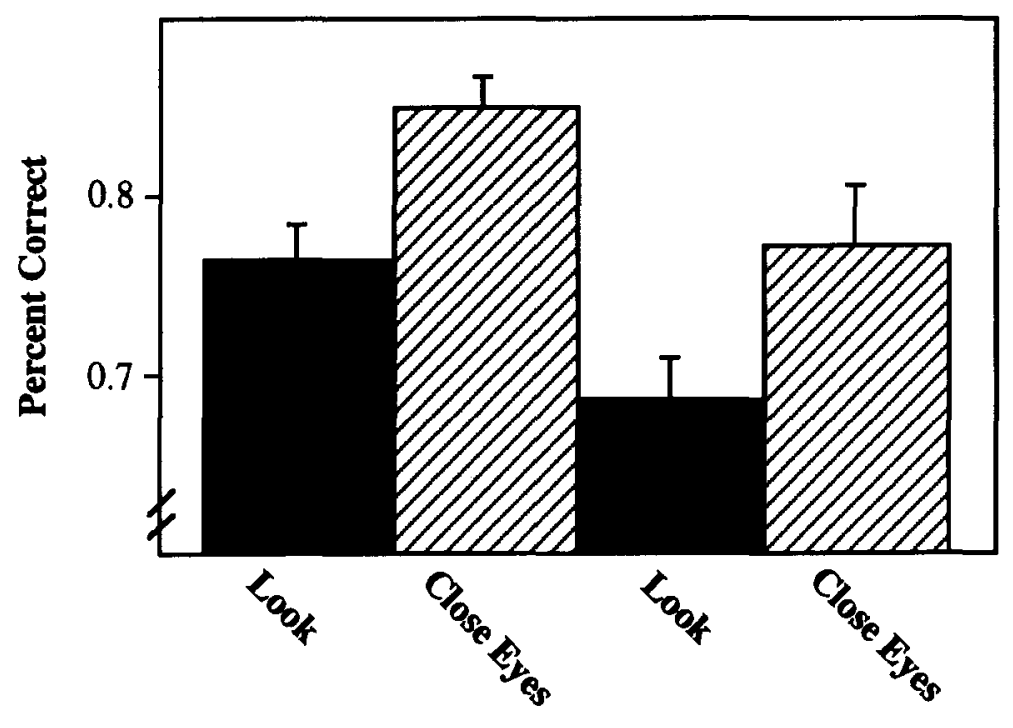

\section{General Knowledge Mathematics}

Figure 3. The data are from Experiment 4. The error bars indicate one standard error of the mean computed for each condition separately. 
Statistically, the only significant effect was that of condition [closed eyes vs. look; $F(1,27)=7.36, M S_{\mathrm{e}}=.03$ ]. ${ }^{4}$

These results demonstrate that averting the gaze has functional consequences. That is, for moderately difficult questions, people are more accurate when they avert their gaze (here, close their eyes) than when they do not. The results appear to contrast with those reported by Ehrlichman (1981). In that experiment, participants answered questions while staring at a video image of the question asker's face ( fixed condition) or were relatively free to look away ( free condition). In the fixed condition, people were faster to begin answering questions than in the free condition, although there was no significant difference between the conditions in number correct. The Ehrlichman study differs from Experiment 4 in several ways that suggest reasons for this failure to find a difference in number correct. In the Ehrlichman study, all participants were in head restraints. This would seem to severely restrict the sorts of gaze aversions possible in the free condition; for example, participants could not turn their heads or cover their eyes as they did frequently in our experiments. In short, the contrast between fixed and free may not have engendered a difference in functional gaze aversion. Second, the phenomenon of gaze aversion seems to be most relevant for tasks of moderate cognitive difficulty. The Ehrlichman study did not include a manipulation of difficulty.

\section{EXPERIMENT 5}

The procedure of Experiment 4 included an odd social situation: looking at the experimenter's nose! We knew from Experiment 3 that gaze aversion was not completely controlled by the social situation, but nonetheless, an odd social situation could create enough distress to reduce performance. To a certain extent, the odd social situation, the distress that accompanies it, and the consequent reduction in performance are consonant with the hypothesis that the function of gaze aversion is to disengage from the environment. That is, because people's faces are typically demanding environmental stimuli, we avert our gaze from them when we need to think hard. This appears to be part of Kendon's (1967) description of the phenomenon that people look at the speaker while listening, but look away when speaking themselves, particularly when answering questions (see Ehrlichman \& Weinberger, 1978). Nonetheless, a demonstration that averting the gaze can be functional in the absence of social distress was in order.

On our account, if current stimulation was simple, there would be little to be gained by averting the gaze. Conversely, if gaze aversion was prevented (as in Experiment 4), it should only cause difficulty when the gaze was directed at a complex stimulus (e.g., a face). We tested that prediction. Participants were shown words for free recall. During the recall period, they were directed to continuously fixate a small cross on a projection screen. In the simple condition, a picture of a sunset across a body of water was projected onto the screen. In the complex condition, a portion of a silent movie was projected onto the screen. We predicted that recall would suffer in the complex condition relative to the simple condition. That is, when participants were unable to avert the gaze (because of the task demands) they would have to cognitively disengage or suppress the environment and that this would be more difficult with the complex scene than with the simple scene.

We knew from other work on free recall of random word lists that not all of the stimuli were equally difficult to recall and that several different strategies might be involved (Glenberg \& Swanson, 1986; Modigliani \& Hedges, 1987). For this reason, we focused our predictions on recall of the midlist items.

\section{Method}

Participants. The 33 participants were students enrolled in introductory psychology classes at the University of Wisconsin, Madison, who earned credit toward a research requirement by participating in the experiment.

Materials and Design. One hundred fifty words were selected from the Toronto Word Pool (Friendly, Franklin, Hoffman, \& Rubin, 1982) and randomly assigned to 10 lists of 15 words each. The order of words within a list was randomized for each subject. Each list was preceded by the word "ready" projected onto a screen approximately $2.5 \mathrm{~m}$ in front of the participant. The ready signal, the words, and the simple and complex recall stimuli were projected over (and centered on) a fixation point affixed to the screen. The ready signal was followed by the presentation of the 15 words, one at a time, projected on the screen for $2 \mathrm{sec}$ each. The list was then followed by a set of 10 three-addend addition problems (e.g., $7+4+6$ ), projected for 2 sec each. Participants were asked to call out the solution to each problem. A 30-sec recall interval followed the last problem. The participants had been instructed to focus on the fixation point throughout the interval, and compliance was checked by the experimenter, who sat on the participant's left. In the simple condition, a slide depicting a sunset was projected onto the screen for the duration of the interval. In the complex condition, a 30-sec segment from the Charlie Chaplin movie Gold Rush was projected without sound. A different segment was projected for each list in the complex condition. In both the simple and the complex conditions, recall was oral, and it was tape recorded for later scoring. Over the course of the 10 lists, each participant viewed 5 lists followed by the simple condition and 5 lists followed by the complex condition. The order of the two conditions was unsystematically mixed and counterbalanced over participants. The participants were given no information regarding the recall condition until after a list had been presented.

Following recall of the 10 th list, the participants were instructed to attempt to recall all lists while keeping their gazes on the fixation point. These data are not reported, because of floor effects.

\section{Results and Discussion}

We treated the first five and last five words in each list as primacy and recency buffers, respectively. Then, for each subject and each list, we computed the proportion recalled of the middle five words. The prediction that recall would be higher in the simple condition $(.28)$ than in 
the complex condition (.23) was confirmed statistically $\left[F(1,32)=7.41, M S_{\mathrm{e}}=.03\right]$. Neither list $(1-5)$ nor the interaction of list and condition was significant.

The results are generally in accord with our predictions: better recall in the simple than in the complex condition. The size of the difference was a bit small from one perspective, although one might also argue that the change from the complex condition to the simple condition was accompanied by an increase of $20 \%$ in amount recalled.

These results confirm that averting the gaze (as in Experiment 4) and not averting the gaze (as in Experiment 5) have functional consequences for memory. The data also disconfirm an alternative interpretation of the results of Experiment 4. As we have noted in the introduction, Kinsbourne's (1972) explanation for LEMs during question answering was that the movements reflect activation of the contralateral cerebral hemisphere. Preventing eye movements in one condition of Experiment 4 (by requiring fixation on the experimenter) might have affected recall because of the effort needed to overcome the putative LEMs. In Experiment 5, fixation was required in both conditions. Thus, any effort needed to overcome LEMs was a constant in the experiment, and any such effort cannot account for the difference between the simple and the complex conditions.

\section{GENERAL DISCUSSION}

In summary, we have shown that people avert their gazes when answering questions of moderate difficulty and that this form of behavior improves performance. Now, why might people avert their gazes, and why might it be beneficial?

An answer to these questions that fits current analyses of memory is that remembering (or problem solving) is at times a dual-task situation: We must engage in remembering while monitoring the environment for unrelated but significant events. If the remembering task is difficult, resources devoted to monitoring the environment are temporarily diverted toward the remembering task. On this account, it is not clear why the eyes would need to be diverted along with cognitive resources. An additional assumption, however, seems warranted. Information-rich components of the environment (e.g., another person) attract attention. Hence, averting the gaze from those components (so that they cannot attract attention) is an activity that facilitates diversion of cognitive resources toward remembering. In fact, this resource-based analysis of gaze aversion is similar to the account offered in Glenberg (1997). On that account, people are normally "clamped" to the environment; that is, conceptualization is controlled by environmental stimulation (cf. Ballard et al., 1997). However, prediction, recollection, and language comprehension all require effortful disengagement from the environment because the environment may be irrelevant to the focus of those activities. Averting the gaze is a relatively cheap way of disengaging from the environment.
There are several differences between the traditional account of a dual-task situation and Glenberg's (1997). The traditional account specifies one or more pools of resources, and tasks interfere to the extent that they draw on and deplete the same pool of resources. The focus of this sort of analysis is often competition between two or more cognitive tasks. For example, in Brooks's (1968) classic experiment, there is evidence for interference between two cognitive tasks that are both spatial and between two cognitive tasks that are both verbal. The account based on clamping and suppression has a different focus: the interplay between environmental control over cognition (when the environment is clamped) and cognitive control over cognition (when the environment is disengaged). Although one might speculate that disengaging the environment draws on a pool of resources, our current hypothesis is that it is more akin to a skill that can be learned for several domains. The data from Experiments 4 and 5 indicate that disengaging from the environment (or failing to do so) can have fairly general effects on cognition. Thus, in Experiment 4, looking at a visual/social stimulus (the experimenter) interferes equally with retrieval of general knowledge and mathematical reasoning. In Experiment 5 , forcing people to keep their eyes on a silent display of actions interferes with the recall of verbal information.

There are at least four reasons why studying gaze aversion and disengaging from the environment may be of more than passing interest. First, the behavior is so frequent as to be characterized as "commonplace" (Kundera, 1996). Second, the results bear a family resemblance to the irrelevant speech effect (LeCompte, 1994) and to the analysis of attentional demands on retrieval (Craik, Govoni, Naveh-Benjamin, \& Anderson, 1996). Third, this sort of behavior has been noted as relevant by investigators in at least two other domains: social behavior (Argyle \& Kendon, 1967), and law enforcement. In the latter context, Fisher and Geiselman (1992) recommend closing the eyes as a component of the cognitive interview designed to facilitate accurate recall of information from eyewitnesses to crimes.

Finally, Glenberg (1997) has proposed that disengaging from the environment may be a significant source of individual differences in cognition. That is, planning, recollective memory, and language all seem to require some ability to disengage from the current environment. If there is reliable variability in the capacity or skill needed to disengage (see Ehrlichman \& Weinberger, 1978, for data on this), this variability ought to be systematically related to the execution of a wide variety of cognitive and behavioral skills.

\section{REFERENCES}

ARGYLE, M., \& Kendon, A. (1967). Experimental analysis of social performance. In L. Berkowitz (Ed.), Advances in experimental social psychology (Vol. 3, pp. 55-98). New York: Academic Press.

Ballakd, D. H. , Hayhoe, M. M., PoOK, P. K., \& RaO, R. P. N. (1997), Deictic codes for the embodiment of cognition. Behavioral \& Brain Sciences, 20, 723-767. 
BrooKs, L. R. (1968). Spatial and verbal components of the act of recall. Canadian Journal of Psychology, 22, 349-368.

Craik, F. I. M., Govoni, R., Naveh-Benjamin, M., \& Anderson, N. D. (1996). The effects of divided attention on encoding and retrieval processes in human memory. Journal of Experimental Psychology: General, 125, 159-180.

DAY, M. E. (1964). An eye movement phenomenon relating to attention, thought and anxiety. Perceptual \& Motor Skills, 19, 443-446.

De Gennaro, L., \& Violani, C. (1988). Reflective lateral eye movements: Individual styles, cognitive and lateralization effects. Neuropsychologia, 26, 727-736.

EhrLichman, H. (1981). From gaze aversion to eye-movement suppression: An investigation of the cognitive interference explanation of gaze patterns during conversation. British Journal of Social Psychology, 20, 233-241.

Ehrlichman, H., \& Weinberger, A. (1978). Lateral eye movements and hemispheric asymmetry: A critical review. Psychological Bulletin, 85, 1080-1101.

Fisher, R. P., \& GEISELMAN, R. E. (1992). Memory enhancing techniques for investigative interviewing: The cognitive interview. Springfield IL: C. C. Thomas.

Friendly, M., Franklin, P. E., Hoffman, D., \& Rubin, D. C. (1982). The Toronto Word Pool: Norms for imagery, concreteness, orthographic variables, and grammatical usage for 1,080 words. Behavior Research Methods \& Instrumentation, 14, 375-399.

GäRDENFORS, P. (1996). Cued and detached representations in animal cognition. Behavioral Processes, 36, 263-273.

GLENBERG, A. M. (1997). What memory is for. Behavioral \& Brain Sciences, 20, 1-19.

Glenberg, A. M., \& Swanson, N. (1986). A temporal distinctiveness account of recency and modality effects. Journal of Experimental Psychology: Learning, Memory, \& Cognition, 12, 3-15.

Kendon, A. (1967). Some functions of gaze-direction in social interaction. Acta Psychologica, 26, 22-63.

KinSBOURNE, M. (1972). Eye and head turning indicates cerebral lateralization. Science, 176, 539-541.

KINSBOURNE, M. (1974). Direction of gaze and distribution of cerebral thought processes. Neuropsychologia, 12, 279-281.

Kundera, M. (1996). Slowness. New York: HarperCollins.

LECOMPTE, D. (1994). Extending the irrelevant speech effect beyond serial recall. Journal of Experimental Psychology: Learning, Memory \& Cognition, 20, 1396-1409.

MESKIN, B. B., \& SingeR, J. L. (1974). Daydreaming, reflective thought. and laterality of eye movements. Journal of Personality \& Social Psychology, 30, 64-71.

Modigliani, V., \& Hedges, D. G. (1987). Distributed rehearsal and the primacy effect in single-trial free recall. Journal of Experimental Psychology: Learning, Memory, \& Cognition, 13, 426-436.

Nelson, T. O., \& NARENS, L. (1980). Norms of 300 general-information questions: Accuracy of recall, latency of recall, and feeling-of-knowing ratings. Journal of Verbal Learning \& Verbal Behavior, 19, 338368.

REDER, L. M., \& RitTeR, E. F. (1992). What determines initial feeling of knowing? Familiarity with question terms, not with the answer. Journal of Experimental Psychology: Learning, Memory, \& Cognition, 18, 435-45t.

RICHARDSON, A. (1978). Subject, task, and tester variables associated with initial eye movement responses. Journal of Mental Imagery, 2, 85-100.

RoEDIGER, H. L., III, GuYnN, M. J., \& Jones, T. C. (1994). Implicit memory: A tutorial review. In G. d'Ydewalle, P. Eelen, \& P. Bertelson (Eds.) International perspectives on psychological science: The state of the art (Vol. 2, pp. 67-94). Hove, U.K.: Erlbaum.

Schwartz, B. L., \& MetCalfe, J. (1992). Cue familiarity but not target retrievability enhance feeling-of-knowing judgments. Journal of Experimental Psychology: Learning, Memory, \& Cognition, 18, 1074-1083.

\section{NOTES}

1. We thank Peter Polson for this suggestion

2 . The correlation between difficulty and gaze aversion for all items is $-.25(p=.23)$. The large reduction in the correlation is due to the nonsignificant, but positive, correlation when the items with difficulties less than .70 are considered. That correlation is $.45(p=.11)$.

3. If one considers just the nine questions analyzed in Experiment 2, the correlation is $-.62(p=.08)$.

4 . The differences between the close eyes and look conditions were generally small and nonsignificant when the easy and difficult items were considered. For the easy items general knowledge items, the difference was -.01 . For the difficult general knowledge items, the difference was $-.09(p>.10)$. For the easy mathematics items, the difference was -.02 . For the difficult mathematics items, the difference was .03 .

(Manuscript received March 14, 1997; revision accepted for publication July 24,1997 .) 